\title{
Learning to Trade Off Between Exploration and Exploitation in Multiclass Bandit Prediction
}

\author{
Hamed Valizadegan \\ Department of Computer \\ Science \\ University of Pittsburgh \\ Pittsburgh, USA \\ hamed@cs.pitt.edu
}

\author{
Rong Jin \\ Department of Computer \\ Science and Engineering \\ Michigan State University \\ East Lansing, PA, USA \\ rongjin@cs.msu.edu
}

\author{
Shijun Wang \\ Radiology and Imaging \\ Sciences \\ National Institute of Health \\ USA \\ wangshi@cc.nih.gov
}

\begin{abstract}
We study multi-class bandit prediction, an online learning problem where the learner only receives a partial feedback in each trial indicating whether the predicted class label is correct. The exploration vs. exploitation tradeoff strategy is a well-known technique for online learning with incomplete feedback (i.e., bandit setup). Banditron [8], a multiclass online learning algorithm for bandit setting, maximizes the run-time gain by balancing between exploration and exploitation with a fixed tradeoff parameter. The performance of Banditron can be quite sensitive to the choice of the tradeoff parameter and therefore effective algorithms to automatically tune this parameter is desirable. In this paper, we propose three learning strategies to automatically adjust the tradeoff parameter for Banditron. Our extensive empirical study with multiple real-world data sets verifies the efficacy of the proposed approach in learning the exploration vs. exploitation tradeoff parameter.
\end{abstract}

\section{Categories and Subject Descriptors}

I.2 [Artificial Intelligence]: Learning

\section{General Terms}

Theory, Algorithms

\section{Keywords}

Online Learning, Bandit Feedback, Multi-class Classification, Exploration vs. Exploitation

\section{INTRODUCTION}

The exploitation vs. exploration tradeoff strategy has been widely used to develop online learning techniques when the feedback provided to the learner is bandit, i.e. the learner only receives the cost of its action but not the cost of other possible actions. Exploration refers to taking an

Permission to make digital or hard copies of all or part of this work for personal or classroom use is granted without fee provided that copies are not made or distributed for profit or commercial advantage and that copies bear this notice and the full citation on the first page. To copy otherwise, to republish, to post on servers or to redistribute to lists, requires prior specific permission and/or a fee.

KDD'11, August 21-24, 2011, San Diego, California, USA.

Copyright 2011 ACM 978-1-4503-0813-7/11/08 ...\$10.00. action that is not recommended by the current model (classifier). It allows the learner to explore the environment (opponent), receive the feedback for different actions, and consequentially gain new knowledge from the environment. Exploitation refers to taking the best action according to the current statistical model in order to maximize the gain. These two objectives are complementary, but opposite: exploration leads to the maximization of the gain in the long run at the risk of losing short term reward, while exploitation maximizes the short term gain at the price of losing the gain over the long run. A careful tradeoff between these two objectives is important to the success of any online learner utilizing the combined strategy [17].

The exploitation vs. exploration tradeoff strategy was originally designed for the multi-armed bandit problem [15] where a slot machine has multiple arms and the player needs to choose the arm with minimum cost (or maximum gain). In each trial, after choosing one arm, the learner receives the cost (award) for the selected arm, but does not know the cost (or award) of the other arms. Under this bandit setting, the learner is required to find the best (possibly mixed) arm in a repeated game. The learner can also be equipped with side information in order to identify the best arm more efficiently. Recently, this technique has been successfully applied to online multi-class classification with partial feedback. Unlike the typical setup of online learning where the learner receives the correct class assignment for an input pattern after making prediction, in online multi-class leaning with partial feedback, the learner only receives an incomplete feedback that indicates if the predicted class is correct. This problem is also referred to as Multiclass Bandit Prediction [8] and has found applications in several domains, including online advertisement and recommender systems [12].

Banditron [8], an algorithm that was recently developed for multi-class bandit prediction, utilizes the exploration vs. exploitation tradeoff technique to handle the challenge of the partial feedback for online multi-class learning. This tradeoff is explicitly captured by a single parameter $\gamma \in(0,0.5)$ in Banditron: with probability $1-\gamma$, the learner will predict the most likely class label based on the current classification model (exploitation), and with probability $\gamma$, the learner will randomly choose one of the remaining class labels for prediction (exploration). Figure 1 shows the performance of Banditron for three different data sets by varying the value of $\gamma$. It is clear that the performance of Banditron strongly depends on the value of $\gamma$ and it is therefore important to develop strategies to automatically tune this parameter. In- 
tuitively, at the beginning of the learning stage, due to the fact that the classification model is trained by a small number of examples, we would expect it to perform poorly. As a result, it may be more desirable to have more exploration and consequentially set $\gamma$ to a large value. As the learning procedure proceeds, after the classification model is updated with sufficiently large number of examples, we expect it to make accurate prediction. As a result, it is desirable to decrease the value of $\gamma$ and consequentially reduce the amount of exploration with increasing number of training examples. Theoretically, as suggested by $[8]^{1}$, the choice of $\gamma=\rho\left(\frac{D K}{t}\right)^{1 / 3}$ minimizes the mistake bound given (i) the complexity measure $D$ of optimal $W$ in the Frobenius norm of $W$, (ii) the number of classes $K$ and (iii) the noise level $\rho^{2}$. One major problem with this choice of $\gamma$ is that it does not depend on the performance of the online classifier, as we would expect a small amount of exploration (i.e., small $\gamma$ ) is needed when the online learner makes accurate prediction. This is because the optimal $\gamma$ is obtained by minimizing the upper bound for the number of mistakes. Since most mistake bounds are concerned with the asymptotical behavior of an online learning algorithm, it tends to be very loose. As a consequence, the resulting $\gamma$ is only optimal in the asymptotical sense, not necessarily for finite horizons. To further support our argument, in Figure 1, we also include the error rates of online multi-class bandit prediction using $\gamma=\rho\left(\frac{D K}{t}\right)^{1 / 3}$ highlighted by horizonal lines. Here, we set $D=2\left|W_{p}\right|_{F}^{2}$ and $\rho=\ell\left(W_{P}\right) / T$ where $W_{p}$ is the classifier learned by the Perceptron ${ }^{3}$. It is clear that the error rates of using this $\gamma$ are far from the true optimals.

This paper is organized as follows. Section 2 reviews the related work to this study. After a short description of Banditron algorithm in Section 3, we propose three different methods to automatically learn the tradeoff parameter $\gamma$ in Sections 3.2, 3.3, and 3.4. Section 4 shows the efficacy of the proposed approaches through an extensive experimental study. Section 5 concludes this work with future directions.

\section{RELATED WORK}

Online multi-class learning in bandit setting has been recently received increasing interests $[9,8,11,2]$. It is closely related to the multi-armed bandit problem [14, 1, 6], where the exploitation vs. exploration tradeoff is used [19, 5, 13]. The multi-armed bandit cannot be applied directly to online multi-class bandit prediction because they do not exploit any side information, such as the feature vector of training examples. Langford et al. extended the multi-armed bandit setting to the case where side information is used to make a better prediction [9]. They refer to the new setting as "contextual bandit problem" and introduced the epoch-greedy algorithm and derived its regret bound. Kakade et al. introduced an efficient algorithm for multiclass bandit prediction that is efficient and guarantees certain regret bound [8], which utilizes the exploitation vs. exploration technique. Wang et al. developed a general potential based framework from which Banditron is a special case [18]. The major problem with Banditron is that its performance could be sensitive

${ }^{1}$ Corollary 3 in [8].

${ }^{2}$ In [8], it is assumed that there exists a $\rho \in(0,1)$ such that $\sum_{i=1}^{T} \ell_{t}(W) \leq \rho T$, where $\ell_{t}(W)$ is a convex loss function.

${ }^{3}$ Assuming that we already know the classifier learned by Perceptron to the parameter that trades off between exploration and exploitation, as shown in Fig 1. To the best of our knowledge, this is the first study that aims to learn the tradeoff parameter for multi-class bandit problem.

\section{OPTIMIZING THE TRADEOFF BETWEEN EXPLORATION AND EXPLOITATION}

\subsection{Preliminaries}

Algorithm 1 shows Banditron [8]. At round $t$, Banditron receives sample $\mathbf{x}_{t}$ and predicts a class label $\widetilde{y}_{t}$ for $\mathbf{x}_{t}$. It then receives an incomplete (bandit) feedback $\left[y_{t}=\widetilde{y}_{t}\right]$, where $\left[y_{t}=\widetilde{y}_{t}\right]$ is an indicator function that outputs 1 if $y_{t}=$ $\widetilde{y}_{t}$ and zero otherwise, and updates its classification model accordingly.

Banditron can be considered as the multi-class perceptron [16] adapted for the bandit setting using the exploitation vs. exploration tradeoff strategy. Similar to the Perceptron algorithm, it computes label $\widehat{y}_{t}$ after receiving an example $x_{t}$ using a linear model $W$. However, due to the bandit feedback, extra (randomization) steps are performed to predict $\widetilde{y}_{t}$ based on the exploitation vs. exploration tradeoff strategy (Steps 7-8): with probability $1-\gamma_{t}$ it predicts class label $\widehat{y}_{t}$ and with probability $\gamma_{t}$ it chooses one random class label for prediction. After receiving the partial feedback $\left[y_{t} \neq \widetilde{y}_{t}\right]$, the algorithm computes the update matrix $x_{t} \delta_{t}$ which, on average, is equivalent to the update matrix used in Perceptron for the full feedback setting. We denote by $M$ the number of mistakes made by algorithm for all the trials, i.e. $M=\sum_{t=1}^{T}\left[\widetilde{y}_{t} \neq y_{t}\right]$.

In our study, we assume a linear model for each class, denoted by $W=\left(\mathbf{w}_{1}, \ldots, \mathbf{w}_{K}\right) \in \mathbb{R}^{d \times K}$, although the extension to nonlinear classifiers using kernel trick is straightforward. We denote by $W_{1}, \ldots, W_{T}$ the sequence of linear classifiers generated by an online learning algorithm over the trials. Our analysis is based on bounding the number of mistakes made by the online learning algorithm when compared to $U$, an arbitrary linear classifier. Since the proposed framework is a stochastic algorithm, we will focus on the expectation of the mistake bound. We denote by $\ell_{t}(W)$ the multi-class hinge loss of classifier $W$ on the training example $\left(\mathbf{x}_{t}, y_{t}\right)$, defined as

$$
\ell_{t}(W)=\ell\left(\max _{k \neq y} \mathbf{w}_{k}^{\top} \mathbf{x}_{t}-\mathbf{w}_{y}^{\top} \mathbf{x}_{t}\right)
$$

where $\ell(z)=\max (0, z+1)$. Theorem 1 gives the mistake bound of the Banditron algorithm that is slightly different from the original work. It provides the foundation for deriving the strategies to learn the tradeoff parameter $\gamma$.

Theorem 1. Let $K$ be the number of classes. After running over a sequence of examples $\mathbf{x}_{1}, \ldots, \mathbf{x}_{T}$, with $\left\|\mathbf{x}_{t}\right\|_{2} \leq 1$ for all $t$, the expected number of mistakes made by Banditron, denoted by $\mathrm{E}[M]$, is bounded as follows

$$
\begin{aligned}
\mathrm{E}[M] & \leq|U|_{F}^{2}+\sum_{t=1}^{T} \ell_{t}(U)+\mathrm{E}\left[\sum_{t=1}^{T} \gamma_{t}\right] \\
& +\mathrm{E}\left\{\sum_{t=1}^{T} \gamma_{t}\left[\widehat{y}_{t}=y_{t}\right]+\sum_{t=1}^{T} \frac{K}{\gamma_{t}}\left[\widehat{y}_{t} \neq y_{t}\right]\right\}
\end{aligned}
$$

where $U$ is any arbitrary weight matrix (classifier) and $\left\{\gamma_{t}\right\}_{t=1}^{T}$ are sampling probabilities of trials. 

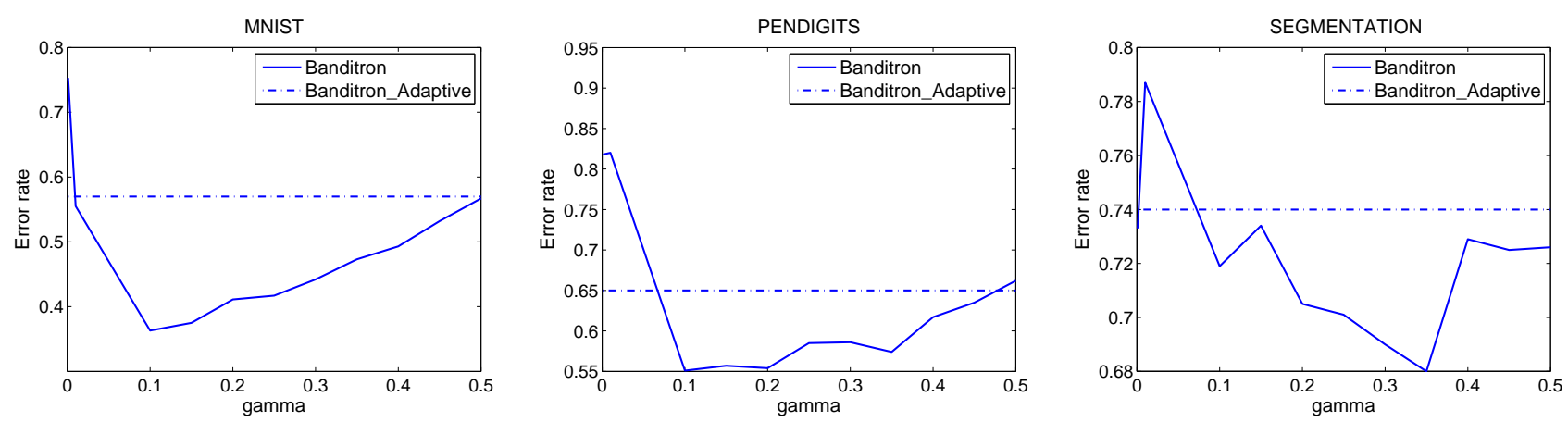

Figure 1: The error rates of Banditron with different choice of $\gamma$ for three different data sets

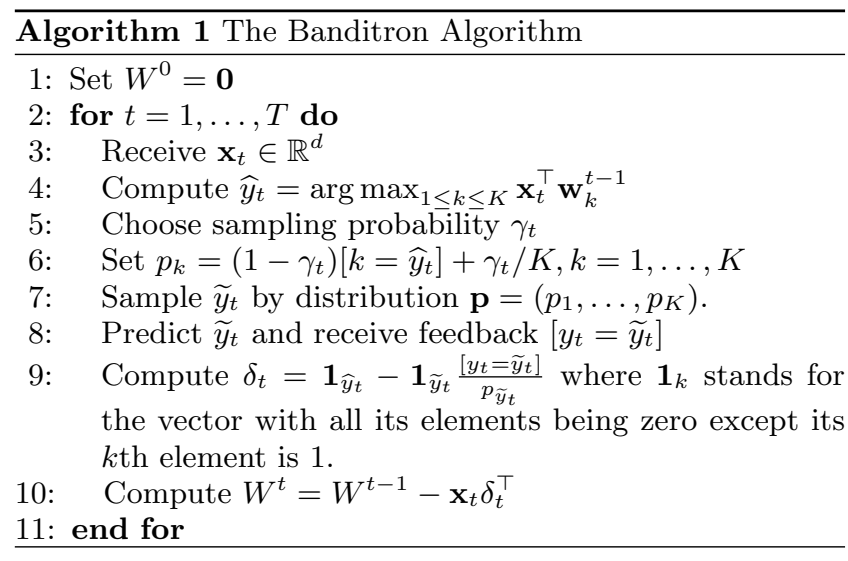

Proof. Following the gradient descent framework for online learning [3], we have the following bound when $\widehat{y}$ is used as the predictor:

$$
\begin{aligned}
& \sum_{t=1}^{T}\left[\widehat{y}_{t} \neq y\right]\left(\ell_{t}\left(W_{t-1}\right)-\ell_{t}(U)\right) \\
\leq & \sum_{t=1}^{T}\left[\widehat{y}_{t} \neq y\right]\left\langle U-W_{t-1}, \nabla \ell_{t}(W)\right\rangle \\
= & \sum_{t=1}^{T}\left\langle U-W_{t-1}, \mathrm{E}_{t}\left[W_{t}-W_{t-1}\right]\right\rangle \\
= & \frac{1}{2} \sum_{t=1}^{T} \mathrm{E}\left[\left|U-W_{t-1}\right|_{F}^{2}-\left|U-W_{t}\right|_{F}^{2}+\left|W_{t}-W_{t-1}\right|_{F}^{2}\right] \\
= & \frac{1}{2}\left|U-W_{0}\right|_{F}^{2}+\mathrm{E}\left[\frac{1}{2} \sum_{t=1}^{T}\left|W_{t-1}-W_{t}\right|_{F}^{2}\right] \\
\leq & \frac{1}{2}|U|_{F}^{2}+\frac{1}{2} \sum_{t=1}^{T} \mathrm{E}\left[\left|\delta_{t}\right|_{F}^{2}\right] \\
\leq & \frac{1}{2}|U|_{F}^{2}+\mathrm{E}\left\{\sum_{t=1}^{T} \gamma_{t}\left[\widehat{y}_{t}=y_{t}\right]+\sum_{t=1}^{T} \frac{K}{\gamma_{t}}\left[\widehat{y}_{t} \neq y_{t}\right]\right\}
\end{aligned}
$$

In the above, the first inequality follows directly the convexity of the loss function, the first equality follows the relationship $\mathrm{E}_{t}\left[\mathbf{x}_{t} \delta_{t}^{\top}\right]=\nabla \ell_{t}(W)$ [18], the second inequality follows $\left|\mathbf{x}_{t}\right|_{2} \leq 1$, and the last inequality follows Lemma 5 of [8].
We complete the proof by using the following relationships

$$
\begin{aligned}
& \sum_{t=1}^{T} \mathrm{E}\left[\widehat{y}_{t} \neq y_{t}\right] \leq \sum_{t=1}^{T}\left[\widehat{y}_{t} \neq y_{t}\right] \ell_{t}(W) \\
& \mathrm{E}[M] \leq \sum_{t=1}^{T} \mathrm{E}\left[\widehat{y}_{t} \neq y_{t}\right]+\sum_{t=1}^{T} \gamma_{t}
\end{aligned}
$$

\section{Remark I:}

By scaling $U$ and $\ell(z)$ as follows

$$
U \rightarrow \eta U, \ell(z) \rightarrow \ell_{\eta}(z)=\max (0, \eta+z)
$$

we generalize Theorem 1 as

$$
\begin{aligned}
\mathrm{E}[M] & \leq \sum_{t=1} t \ell_{t}(U)+\mathrm{E}\left[\sum_{t=1}^{T} \gamma_{t}\right] \\
& +\frac{\eta}{2}|U|_{F}^{2}+\frac{1}{\eta} \mathrm{E}\left\{\sum_{t=1}^{T} \gamma_{t}\left[\widehat{y}_{t}=y_{t}\right]+\sum_{t=1}^{T} \frac{K}{\gamma_{t}}\left[\widehat{y}_{t} \neq y_{t}\right]\right\}
\end{aligned}
$$

By minimizing over scaling factor $\eta$, we have

$$
\begin{aligned}
& \mathrm{E}[M] \leq \sum_{t=1}^{T} \ell_{t}(U)+\mathrm{E}\left[\sum_{t=1}^{T} \gamma_{t}\right] \\
& +|U|_{F} \sqrt{2 \mathrm{E}\left\{\sum_{t=1}^{T} \gamma_{t}\left[\widehat{y}_{t}=y_{t}\right]+\sum_{t=1}^{T} \frac{K}{\gamma_{t}}\left[\widehat{y}_{t} \neq y_{t}\right]\right\}}
\end{aligned}
$$

which the same inequality used by [8] to obtain the mistake bound.

Given the bound provided in Theorem 1, the optimal set of sampling probabilities $\left\{\gamma_{t}\right\}_{t=1}^{T}$ will be obtained by minimizing the mistake bound stated in Theorem 1, i.e.

$$
\mathcal{L}=\sum_{t=1}^{T} \frac{K}{\gamma_{t}}\left[\widehat{y}_{t} \neq y_{t}\right]+\sum_{t=1}^{T} \gamma_{t}\left(1+\left[\widehat{y}_{t}=y_{t}\right]\right)
$$

However $\left[\widehat{y}_{t} \neq y_{t}\right]$ and $\left[\widehat{y}_{t}=y_{t}\right]$ are not provided explicitly in the partial feedback, making it impossible to obtain $\gamma_{t}$ by directly minimizing the upper bound. Note that in the previous studies, this difficulty is resolved by bound $\left[\widehat{y}_{t}=\right.$ $\left.y_{t}\right] \leq 1$, leading to the choice of $\gamma_{t}$ independent from the prediction performance of the online classifier. To address the difficulty, we approximate $\left[\widehat{y}_{t} \neq y_{t}\right]$ and $\left[\widehat{y}_{t}=y_{t}\right]$ by two biased estimators $\mu_{t}$ and $\tau_{t}$, respectively: 
- Approximate $\left[\widehat{y}_{t}=y_{t}\right]$ by $\mu_{t}=2\left[\widehat{y}_{t}=\widetilde{y}_{t}\right]\left[\widetilde{y}_{t}=y_{t}\right]$. This is because

$$
\begin{aligned}
{\left[\widehat{y}_{t}=y_{t}\right] } & =\mathrm{E}_{t}\left[\frac{\left[\widehat{y}_{t}=\widetilde{y}_{t}\right]\left[\widetilde{y}_{t}=y_{t}\right]}{p_{\widetilde{y}_{t}}}\right] \\
& \leq \mathrm{E}_{t}\left[\frac{\left[\widehat{y}_{t}=\widetilde{y}_{t}\right]\left[\widetilde{y}_{t}=y_{t}\right]}{1-\gamma_{t}}\right] \\
& \leq \mathrm{E}_{t}\left[\mu_{t}\right]
\end{aligned}
$$

Note that in the last step, we use the fact $\gamma_{t} \leq 1 / 2$.

- Approximate $\left[\widehat{y}_{t} \neq y_{t}\right]$ by $\tau_{t}=1-\left[\widehat{y}_{t}=\widetilde{y}_{t}\right]\left[\widetilde{y}_{t}=y_{t}\right]$

$$
\begin{aligned}
{\left[\widehat{y}_{t} \neq y_{t}\right] } & =1-\left[\widehat{y}_{t}=y_{t}\right] \\
& \leq 1-\mathrm{E}_{t}\left[\left[\widehat{y}_{t}=\widetilde{y}_{t}\right]\left[\widetilde{y}_{t}=y_{t}\right]\right] \\
& =\mathrm{E}_{t}\left[\tau_{t}\right]
\end{aligned}
$$

Using $\mu_{t}$ and $\tau_{t}$ allows us to compute the two quantities without have to know $\left[\widehat{y}_{t}=y_{t}\right]$ or $\left[\widehat{y}_{t} \neq y_{t}\right]$. However, it is infeasible to use $\mu_{t}$ and $\tau_{t}$ for approximation because both of them require knowing $y_{t}$. We address this difficulty by approximating $\mu_{t}$ and $\tau_{t}$ with the average of $\mu$ and $\tau$ over all the trials up to the time $t$, i.e., replacing $\mu_{t}$ and $\tau_{t}$ with $\sum_{i=1}^{t-1} \mu_{i} /(t-1)$ and $\sum_{i=1}^{t-1} \tau_{i} /(t-1)$, respectively. Combining with the trivial bounds that $\left[\widehat{y}_{t}=y_{t}\right] \leq 1$ and $\left[\widehat{y}_{t} \neq y_{t}\right] \leq 1$, in the following subsections, we consider three strategies for approximating $\left[\widehat{y}_{t}=y_{t}\right]$ and $\left[\widehat{y}_{t} \neq y_{t}\right]$

- $\left[\widehat{y}_{t} \neq y_{t}\right] \leq \tau_{t}$ and $\left[\widehat{y}_{t}=y_{t}\right] \leq \mu_{t}$

- $\left[\widehat{y}_{t} \neq y_{t}\right] \leq 1$ and $\left[\widehat{y}_{t}=y_{t}\right] \leq \mu_{t}$

- $\left[\widehat{y}_{t}=y_{t}\right] \leq 1$ and $\left[\widehat{y}_{t} \neq y_{t}\right] \leq \tau_{t}$

In order to generate a general family of functions for $\gamma_{t}$, we introduce the concept of good support function, which is the key gradient for defining the tradeoff parameter.

Definition 2. A function $\omega(z)$ defined in the domain of $z \geq 0$ is called a good support function if it satisfies the following conditions: (a) $\omega(z)$ is concave for $z \geq 0$ and $\omega(0) \geq 0,(b) \omega(z)$ is monotonically increasing, i.e., $\omega^{\prime}(z)>0$, for $z \geq 0$, (c) $\omega(z)$ is Lipschitz continuous with Lipschitz constant L, i.e., $\omega^{\prime}(z) \leq L$, for $z \geq 0$, and (d) there exists a constant $\rho \geq 1$ such that for any $t \geq 0$ and $z \geq 0$, we have $\omega^{\prime}(z) \leq \rho^{t} \omega^{\prime}(z+t)$.

The discussion below allows us to connect the definition of good support function to the desired properties of $\gamma_{t}$.

- Given Property (a), it is easy to conclude that $\omega^{\prime}(z)$ is a non-decreasing function of $z, \omega^{\prime}(z+t) \leq \omega^{\prime}(z)$ or $\frac{1}{\omega^{\prime}(z)} \leq \frac{1}{\omega^{\prime}(z+t)}$ for $t \geq 0$. This non-increasing property of $\omega^{\prime}(z)$ also allows us to define non-increasing $\gamma_{t}$ that is bounded between 0 and $1 / 2$.

- Property (b) allows us to have $\omega(z) \leq \omega(z+t)$ or $\frac{1}{\omega(z+t)} \leq \frac{1}{\omega(z)}$ for $t \geq 0$, another important quantity for deriving the mistake bound.

- Property (c) and (d) allow us to upper-bound $\omega^{\prime}(z)$ by a constant $L$ and by $\omega^{\prime}(z+t)$ for $t \geq 0$.

The following proposition gives examples of good support function.
Proposition 1. (1) $\omega(z)=(a+z)^{\lambda}$, with $\lambda \in(0,1]$ and $a>0$, is a good support function, with Lipschitz constant $L=\lambda a^{\lambda-1}$, and $\rho=e^{(1-\lambda) / a}$, and (2) $\omega(z)=\ln (a+z)$ with $a>0$ is a good support function, with $L=1 / a$, and $\rho=e^{1 / a}$.

Proof. We only show the result for $\omega(z)=(a+z)^{\lambda}$. A similar derivation can be applied to $\omega(z)=\ln (a+z)$. For Lipschitz continuous constant $L$, we have

$$
L=\max _{z \geq 0} \frac{\lambda}{(a+z)^{1-\lambda}}=\lambda a^{\lambda-1}
$$

For $\rho$, we have

$$
\frac{(a+z+t)^{1-\lambda}}{(a+z)^{1-\lambda}} \leq(1+t / a)^{1-\lambda} \leq e^{t(1-\lambda) / a}
$$

leading to $\rho=e^{(1-\lambda) / a}$.

\subsection{Finding Optimal $\gamma$ using $\mu_{t}$ and $\tau_{t}$}

Using $\left[\widehat{y}_{t}=y_{t}\right] \leq \mu_{t}$ and $\left[\widehat{y}_{t} \neq y_{t}\right] \leq \tau_{t}$, we approximate $\mathcal{L}$ by

$$
\widehat{\mathcal{L}}=\sum_{t=1}^{T} \frac{K}{\gamma_{t}} \tau_{t}+\sum_{t=1}^{T} \gamma_{t}(\mu+1) .
$$

To bound $\widehat{\mathcal{L}}$, we introduce two good support functions $\omega_{1}(z)$ and $\omega_{2}(z)$, with $\omega_{1}^{\prime}(z) \geq \omega_{2}^{\prime}(z)$ for any $z \geq 0$, and define $\gamma_{t}$ as follows

$$
\gamma_{t}=\frac{\omega_{2}^{\prime}\left(\sum_{i=1}^{t-1} 1+\mu_{i}\right)}{2 \omega_{1}^{\prime}\left(\sum_{i=1}^{t-1} \tau_{i}\right)}
$$

We have $\gamma_{t} \leq 1 / 2$ for any $t$ because

$$
\gamma_{t} \leq \frac{\omega_{1}^{\prime}\left(\sum_{i=1}^{t-1} 1+\mu_{i}\right)}{2 \omega_{1}^{\prime}\left(\sum_{i=1}^{t-1} \tau_{i}\right)} \leq \frac{\omega_{1}^{\prime}\left(\sum_{i=1}^{t-1} \tau_{i}\right)}{2 \omega_{1}^{\prime}\left(\sum_{i=1}^{t-1} \tau_{i}\right)}=\frac{1}{2}
$$

The first inequality follows $\omega_{1}^{\prime}(z) \geq \omega_{2}^{\prime}(z)$, and the last step follows the property $\tau_{i} \leq 1$ and the fact that $\omega_{1}^{\prime}(z)$ is nonincreasing in $z$. In addition, since $\omega_{1}^{\prime}(z)$ and $\omega_{2}^{\prime}(z)$ are nonincreasing functions of $z, \gamma_{t}$ is decreasing in $\mu_{t}$ and increasing in $\tau_{t}$, implying that the algorithm gives a large amount of exploration when the classifier makes a number of mistakes, and a small amount of exploration when the classifier encounters only a few prediction errors. The following proposition shows key properties for $\gamma_{t}$ in Equation 4.

Proposition 2. Given $\gamma_{t}$ defined in (4), we have the following inequalities:

$$
\begin{aligned}
\sum_{t=1}^{T} \gamma_{t} & \leq \frac{\rho_{2} \omega_{2}(T)}{2 \omega_{1}^{\prime}\left(\sum_{t=1}^{T} \tau_{t}\right)} \\
\sum_{t=1}^{T} \gamma_{t} \mu_{t} & \leq \frac{\rho_{2}^{2} \omega_{2}\left(\sum_{t=1}^{T} \mu_{t}\right)}{2 \omega_{1}^{\prime}\left(\sum_{t=1}^{T} \tau_{t}\right)} \\
\sum_{t=1}^{T} \frac{K}{\gamma_{t}} \mu_{t} & \leq \frac{2 K \rho_{1} \omega_{1}\left(\sum_{t=1}^{T} \tau_{t}\right)}{\omega_{2}^{\prime}\left(\sum_{t=1}^{T} 1+\mu_{t}\right)}
\end{aligned}
$$

where $\rho_{1}$ and $\rho_{2}$ are the constants defined respectively for the good support functions $\omega_{1}$ and $\omega_{2}$. 
Proof. Define $A_{t}=\sum_{i=1}^{t} \tau_{i}$. We have

$$
\begin{aligned}
\sum_{t=1}^{T} \frac{K}{\gamma_{t}} \tau_{t} & =\sum_{t=1}^{T} \frac{2 K \omega_{1}^{\prime}\left(A_{t-1}\right) \tau_{t}}{\omega_{2}^{\prime}\left(\sum_{i=1}^{t-1} 1+\mu_{i}\right)} \\
& =\sum_{t=1}^{T} \frac{2 K \omega_{1}^{\prime}\left(A_{t-1}\right)\left(A_{t}-A_{t-1}\right)}{\omega_{2}^{\prime}\left(\sum_{i=1}^{t-1} 1+\mu_{i}\right)} \\
& \leq \frac{2 K \sum_{t=1}^{T} \omega_{1}^{\prime}\left(A_{t-1}\right)\left(A_{t}-A_{t-1}\right)}{\omega_{2}^{\prime}\left(\sum_{i=1}^{T} 1+\mu_{i}\right)}
\end{aligned}
$$

where the inequality follows that $\omega_{2}^{\prime}$ is a non-increasing function. Since

$$
\begin{aligned}
\sum_{t=1}^{T} \omega_{1}^{\prime}\left(A_{t-1}\right)\left(A_{t}-A_{t-1}\right) & \leq \rho_{1} \sum_{t=1}^{T} \omega_{1}^{\prime}\left(A_{t}\right)\left(A_{t}-A_{t-1}\right) \\
& \leq \rho_{1} \sum_{t=1}^{T} \omega_{1}\left(A_{t}\right)-\omega_{1}\left(A_{t-1}\right) \\
& \leq \rho_{1} \omega_{1}\left(A_{T}\right),
\end{aligned}
$$

we have the first inequality. In the above, the first step follows the definition of good support functions (i.e. $\omega_{1}^{\prime}\left(A_{t-1}\right) \leq$ $\left.\rho_{1} \omega_{1}^{\prime}\left(A_{t}\right)\right)$, the second step is due to the concavity of $\omega_{1}$ and the last step is due to $\omega_{1}(0) \geq 0$. Combining the above results produces the first inequality in the proposition. Similar to the first inequality, for the second inequality, we define $B_{t}=\sum_{i=1}^{t} \mu_{i}$ and have

$$
\begin{aligned}
\sum_{t=1}^{T} \gamma_{t} \mu_{t} & =\sum_{t=1}^{T} \frac{\omega_{2}^{\prime}\left(B_{t-1}+t-1\right)}{2 \omega_{1}^{\prime}\left(A_{t-1}\right)} \mu_{t} \\
& \leq \frac{\sum_{t=1}^{T} \omega_{2}^{\prime}\left(B_{t-1}\right)\left(B_{t}-B_{t-1}\right)}{2 \omega_{1}^{\prime}\left(A_{T}\right)} \\
& \leq \frac{\sum_{t=1}^{T} \rho_{2}^{2} \omega_{2}^{\prime}\left(B_{t}\right)\left(B_{t}-B_{t-1}\right)}{2 \omega_{1}^{\prime}\left(A_{T}\right)} \\
& \leq \frac{\rho_{2}^{2} \omega_{2}\left(B_{T}\right)}{2 \omega_{1}^{\prime}\left(A_{T}\right)}
\end{aligned}
$$

The second equality follows $\omega_{2}^{\prime}\left(B_{t-1}\right) \leq \rho_{2}^{\mu_{t}} \omega_{2}^{\prime}\left(B_{t}\right)$ and $\mu_{t} \leq$ 2. The same analysis is applied to show the last inequality.

THEOREM 3. Let $\omega_{1}(z)$ and $\omega_{2}(z)$ be two good support functions with $\omega_{1}^{\prime}(z) \geq \omega_{2}^{\prime}(z)$ for any $z \geq 0$. By running Algorithm 1 with $\gamma_{t}$ set as in Eq. (4), we have the following bound for the expected number of misclassified examples:

$$
\begin{aligned}
\mathrm{E}[M] & \leq \sum_{t=1}^{T} \ell_{t}(U)+\frac{\rho_{2} \omega_{2}(T)}{2 \omega_{1}^{\prime}(T)} \\
& +|U|_{F}\left(\sqrt{\frac{2 \rho_{1} K \omega_{1}(3 T)}{\omega_{2}^{\prime}(3 T)}}+\rho_{2} \sqrt{\frac{\omega_{2}(2 T)}{2 \omega_{1}^{\prime}(2 T)}}\right)
\end{aligned}
$$

where $\rho_{1}$ and $\rho_{2}$ are the constants of two good support functions.

PROOF. The proof is straightforward by using the result in Remark I, Proposition 2, inequality $\sqrt{a+b} \leq \sqrt{a}+\sqrt{b}$, and the properties of good support functions.

Corollary 4. Suppose $\gamma_{t}$ is in Eq. (4) with $\omega_{1}(z)=(1+$ $z)^{\lambda_{1}}$ and $\omega_{2}(z)=(1+z)^{\lambda_{2}}$, where $\lambda_{1}, \lambda_{2} \in(0,1]$ and $\lambda_{1}=$ $\lambda_{2}+1 / 3$. By running Algorithm 1, we have the following bound for the expected number of misclassified examples:

$$
\begin{aligned}
\mathrm{E}[M] & \leq \sum_{t=1}^{T} \ell_{t}(U)+\frac{\rho_{2}}{\lambda_{2}+\frac{1}{3}}(1+T)^{\frac{2}{3}} \\
& +|U|_{F}\left(\sqrt{\frac{\rho_{2} e^{\frac{1}{3}} K}{\lambda_{2}}}(1+3 T)^{\frac{2}{3}}+\frac{\rho_{2}}{\sqrt{\lambda_{2}+\frac{1}{3}}}(1+2 T)^{\frac{1}{3}}\right)
\end{aligned}
$$

where $\rho_{2}=e^{1-\lambda_{2}}$. This bound is of $O\left(T^{2 / 3}\right)$ and similar to the bound of the original Banditron.

\section{Remark II:}

Although the mistake bounds given in Theorem 3 and Corollary 4 are not better than the result derived in the previous study, we emphasize that the analysis of mistake bound is often loose because its focus is to obtain the asymptotical behavior of the learner. The key advantage of the tradeoff parameter $\gamma_{t}$ defined in Eq. 4 is its capability of adapting to the classification performance (i.e., $\mu_{t}$ and $\tau_{t}$ ) of the learner, making it intuitively more attractive.

\subsection{Finding Optimal $\gamma$ using $\mu_{t}$}

Using $\left[\widehat{y}_{t} \neq y_{t}\right] \leq 1$ and $\left[\widehat{y}_{t}=y_{t}\right] \leq \mu_{t}$, we upper bound $\mathcal{L}$ by

$$
\widehat{\mathcal{L}}=\sum_{t=1}^{T} \frac{K}{\gamma_{t}}+\sum_{t=1}^{T} \gamma_{t}\left(1+\mu_{t}\right)
$$

Given a good support function $\omega(z)$, we define $\gamma_{t}$ as

$$
\gamma_{t}=\frac{1}{2 L} \omega^{\prime}\left(\sum_{i=1}^{t-1} 1+\mu_{i}\right)
$$

It is straightforward to see $\gamma_{t} \in[0,1 / 2]$. Since $\omega(z)$ is a concave function, $\omega^{\prime}(z)$ is a non-increasing function of $z$, leading to a decreasing value for $\gamma_{t}$ as more and more training examples have been classified correctly. The following proposition shows key properties for $\gamma_{t}$ in (5).

Proposition 3. Given $\gamma_{t}$ defined in (5), we have the following inequalities:

$$
\begin{aligned}
\sum_{t=1}^{T} \gamma_{t} \mu_{t} & \leq \frac{\rho^{2}}{2 L} \omega\left(\sum_{t=1}^{T} \mu_{t}\right) \\
\sum_{t=1}^{T} \gamma_{t} & \leq \frac{\rho}{2 L} \omega(T) \\
\sum_{t=1}^{T} \frac{K}{\gamma_{t}} & \leq \frac{2 K L T}{\omega^{\prime}\left(\sum_{t=1}^{T} 1+\mu_{t}\right)}
\end{aligned}
$$

The proof is almost identical to that for Proposition 2.

TheOREM 5. Let $\omega(z)$ be a good support function. By running Algorithm 1 with $\gamma_{t}$ set as in Eq. (5), we have the following bound for the expected number of mistakes made by the algorithm:

$$
\mathrm{E}[M] \leq \sum_{t=1}^{T}+\frac{\rho \omega(T)}{2 L}+|U|_{F}\left(\rho \sqrt{\frac{\omega(2 T)}{2 L}}+\sqrt{\frac{2 K L T}{\omega^{\prime}(3 T)}}\right)
$$


The following corollary directly follows from Proposition 1 and Theorem 5.

Corollary 6. By running Algorithm 1 with $\gamma_{t}$ as in Eq. (5) and $\omega(z)=(1+z)^{\lambda}$, where $\lambda \in(0,1]$, we have the following bound for the expected number of classification mistakes:

$$
\begin{aligned}
\mathrm{E}[M] & \leq \sum_{t=1}^{T} \ell_{t}(U)+\frac{e^{1-\lambda}}{2 \lambda}(1+T)^{\lambda} \\
& +|U|_{F}\left(\frac{e^{1-\lambda}}{\sqrt{2 \lambda}}(1+2 T)^{\frac{\lambda}{2}}+\sqrt{2 K}(1+T)^{\frac{1-\lambda}{2}} T^{\frac{1}{2}}\right)
\end{aligned}
$$

When $\lambda=2 / 3$, we have $\mathrm{E}[M]=O\left(T^{2 / 3}\right)$ which is the same convergence rate as Banditron.

\subsection{Finding Optimal $\gamma$ using $\tau_{t}$}

Similar to the approach presented in the previous sections, we set $\gamma_{t}$ as

$$
\gamma_{t}=\frac{\omega_{2}^{\prime}(t)}{2 \omega_{1}^{\prime}\left(\sum_{i=1}^{t-1} \tau_{i}\right)}
$$

where $\omega_{1}(z)$ and $\omega_{2}(z)$ are two good support functions and $\omega_{1}^{\prime}(z) \geq \omega_{2}^{\prime}(z)$. It is easy to verify that $\gamma_{t} \in(0,1 / 2]$ due to the properties of a good support function. The proposition below allows us to bound $\sum_{t=1}^{T} \gamma_{t}$ and $\sum_{t=1}^{T} K / \gamma_{t}$.

Proposition 4. Given the construction of $\gamma_{t}$ in (6), we have the following inequalities:

$$
\begin{aligned}
\sum_{t=1}^{T} \frac{K}{\gamma_{t}} \tau_{t} & \leq 2 K \rho \frac{\omega_{1}\left(\sum_{t=1}^{T} \tau_{t}\right)}{\omega_{2}^{\prime}(T)} \\
\sum_{t=1}^{T} \gamma_{t} & \leq \frac{\omega_{2}(T)}{2 \omega_{1}^{\prime}\left(\sum_{t=1}^{T} \tau_{t}\right)}
\end{aligned}
$$

Proof. Similar to Proposition 2 and 3.

ThEOREM 7. Let $\omega_{1}(z)$ and $\omega_{2}(z)$ be two good support functions. By running Algorithm 1 with $\gamma_{t}$ set as in Eq. (6), we have the following bound for the number of misclassified examples $M=\sum_{t=1}^{T}\left[\widehat{y}_{t} \neq y_{t}\right]$ :

$$
\begin{aligned}
\mathrm{E}[M] & \leq \sum_{t=1}^{T} \ell_{t}(U)+\frac{\omega_{2}(T)}{2 \omega_{1}^{\prime}\left(\sum_{t=1}^{T} \tau_{t}\right)} \\
& +|U|_{F}\left(\sqrt{\frac{\omega_{2}(T)}{2 \omega_{1}^{\prime}(T)}}+\sqrt{2 K \rho_{1} \frac{\omega_{1}(T)}{\omega_{2}^{\prime}(T)}}\right)
\end{aligned}
$$

Proof. The proof directly follows Theorem 1 and Proposition 4 .

The following corollary directly follows from the result of Proposition 1 and Theorem 7.

Corollary 8. By running Algorithm 1 with $\gamma_{t}$ in Eq. (6) and $\omega_{1}=(1+z)^{\lambda_{1}}$ and $\omega_{2}=(1+z)^{\lambda_{2}}$ with $\lambda_{1}, \lambda_{2} \in(0,1]$, we have the following bound for the expected number of misclassified examples

$$
\begin{aligned}
\mathrm{E}[M] & \leq \sum_{t=1}^{T} \ell_{t}(U)+\frac{1}{2 \lambda_{1}}(1+T)^{\lambda_{2}+1-\lambda_{1}} \\
& +|U|_{F}\left(\sqrt{\frac{1}{2 \lambda_{1}}}(1+T)^{\frac{\lambda_{2}+1-\lambda_{1}}{2}}+\sqrt{\frac{2 k e^{1-\lambda_{1}}}{\lambda_{2}}}(1+T)^{\frac{\lambda_{1}+1-\lambda_{2}}{2}}\right)
\end{aligned}
$$

with $\lambda_{1}=\lambda_{2}+\frac{1}{3}$ we have $E[M]=O\left(T^{2 / 3}\right)$ which is of the same rate as Banditron.

\section{EXPERIMENTS}

In this section, we conduct experiments on several realword data sets to validate the proposed strategies for balancing the tradeoff between exploration and exploitation.

\subsection{Data Set}

Several real-world data sets from UCI data repository [7] and LIBSVM web page [4] are used in our study as described in the following. Notice that for some of these data sets, there were two separate sets, one for training and one for testing. We only used the training set in our experiments.

- RCV1. RCV1 consists of 15,564 of training documents for text categorization. Each document is a news report from Reuters [10], represented by a vector of 47,236 dimension, and belongs to one of the 53 classes.

- MNIST. MNIST contains grey scale images of size $28 \times 28$ for hand written digits. It contains 60000 training samples, each represented by 780 features.

- Protein. Protein has 17766 samples, represented by 357 features and three classes.

- Letter. Letter contains 15000 instances of 26 characters, represented by 16 features.

- optdigits. This is a normalized bitmaps of handwritten digits from 30 people that contains 3823 instances, each represented by $8 \times 8$ features.

- pendigits. This is another digits collection containing 7495 samples, each with 16 features.

- Nursery. Originally developed to rank applications for nursery school, it has 12960 records, each represented by 8 features belonging to one of 4 classes (we removed one class that only had two samples).

- Isolet. Isolet contains 7797 spoken alphabet with 617 attributes and 26 classes.

- Abalone. Developed to predict the age of abalone from physical measurement, it contains 4177 records, each represented by 8 features and one of the 29 classes.

- Car. This data set contains 1728 samples, each represented by 6 features and one of the four classes.

- Waveform. Waveform data set contains 5000 samples of three classes, each represented by 21 features.

- RedWineQuality. This data set contains 1599 red wine records, each represented by 11 features and one of the 6 classes.

- RedWineQuality. WhiteWineQuality contains 4898 white wine records, each represented by 11 features and one of the 7 classes.

- Segmentation. The image Segmentation data set contains 2310 samples. Each sample is represented by 19 features and belongs to one of the 7 classes. 

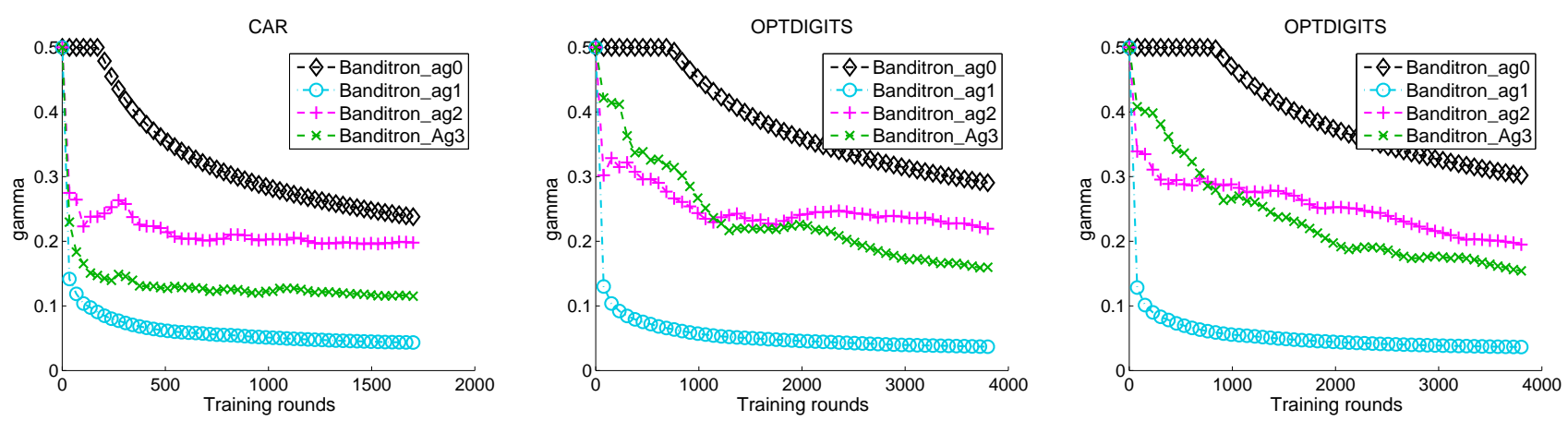

Figure 2: Behavior of $\gamma$ in different methods for data sets, Car and Optdigits. There are two figures for Optdigits to stress that the proposed methods adapt $\gamma$ based on the sequence of the samples over trials.

\subsection{Experimental Settings}

We refer to the algorithms developed in Sections 3.2, 3.3, and 3.4 as Banditron_ag3, Banditron_ag1 and Banditron _ag2. To evaluate the classification performance of the three proposed learning strategies for exploitation vs. exploration tradeoff parameter $\gamma$, we compare them with three different version of Banditron, namely, Banditron_Worst, Banditron_Best, and Banditron_ag0. Banditron_Worst and Banditron_Best are Banditron algorithm when $\gamma$ is set to the worst and best value for a given data. The worst and best $\gamma$ are found from the predefined set $S=\{0.001,0.01,0.1$, $0.15,0.2,0.25,0.3,0.35,0.4,0.45,0.5\}$. Banditron_ag0 is the Banditron with the adaptive $\gamma_{t}=\min \left(0.5, \rho\left(\frac{D K}{t}\right)^{1 / 3}\right)$ as suggested in [8] for the general agnostic case. We used $D=2\left|W_{p}\right|_{F}^{2}$ and $\rho=\ell\left(W_{P}\right) / T$ where $W_{p}$ is the classifier learned by the Perceptron ${ }^{4}$. We repeat each experiment 50 times by generating random sequences of instances and report the average accumulate error rates, which are computed as the ratio of the number of misclassified samples to the number of samples received so far. For all three proposed methods in all the experiments, we use similar good support functions $\omega(z)=\omega_{1}(z)=\omega_{2}(z)=(1+z)^{\lambda}$ with $\lambda=0.1$ for a fair comparison. Also notice that the result is stable for most of these data sets with different values of $\lambda$.

\subsection{Experimental results}

To study the behavior of different learning algorithms over trials, we show the average error rates of all the methods over the entire online process in Figure 3. First notice that there is big gap between Banditron_worst and Banditron_Best in all data sets that emphasizes that the Banditron algorithm can perform very poorly if $\gamma$ is not set appropriately.

We observe that overall the proposed algorithms exhibit similar or better learning rates as the Banditron algorithm with the optimal $\gamma$. In particular, banditron_ag2 and banditron $\_$ag3 yields the best performance among the algorithms in comparison. In most of the data sets, banditron_ag2 and banditron_ag3 perform significantly better than banditron_ag0 which suggests that $\gamma_{t}=\min \left(0.5, \rho\left(\frac{D K}{t}\right)^{1 / 3}\right)$ is not a good adaptive choice (even with the assumption that Perceptron setting for variables $D$ and $\rho$ is known). As a few examples, notice that the final error rate of banditron_ag0 is $56 \%$ versus $37 \%$ error rate of banditron_ag2, banditron_ag3

\footnotetext{
$\overline{{ }^{4} \text { We also tried } \gamma_{t}=0.5 t^{-1 / 3}}$. The proposed approach consistently produces better result than $\gamma_{t}=0.5 t^{-1 / 3}$ as well.
}

and banditron_best for MNIST data set. For Optdigits data set, the final error rate of banditron_ag2 and banditron_ag3 is $56 \%$ which is significantly low compared to $59 \%$ error rate of banditron_best and banditron_ag0. The latter example also suggests that our adaptive strategy is better than the Banditron with a single best $\gamma$.

Although better than banditron_worst, the performance of banditron_ag1 is not comparable to that of the other methods for most of the cases. This can be explained by the inherited difference between banditron_ag1 and the other two proposed approaches. Unlike banditron_ag2 and banditron_ag3 where two good support functions are introduced to determine $\gamma_{t}$, the $\gamma_{t}$ defined in banditron_ag1 is determined by a single good support function. As a result, we have a better control of the value for $\gamma$ over time in banditron ag2 2 and banditron_ag3 by a trade-off between two functions: one which is the decreasing function of time and the other which is the increasing function of the number of misclassified examples.

Figure 2 shows how $\gamma$ changes during the learning process for different algorithms for two data sets, car and optdigits. We also plot two different runs of optdigits to see how the proposed methods is sensitive to the order of presented samples. Notice that unlike banditron_ag0 that constantly decreases the value of $\gamma$, the proposed methods are more adaptive. More particularly, banditron_ag2 and banditron_ag3 update $\gamma$ based on the performance of Banditron over trials, and if needed, they increase the value of $\gamma$. Moreover, the proposed methods are sensitive to the sequence of the samples, and chooses the best updating rule based on how samples presented over trials and how much the learning process in Banditron succeeded so far.

\section{CONCLUSION}

In this work, we study the problem of optimizing the exploration-exploitation tradeoff in the context of multi-class bandit prediction. We proposed three different strategies to automatically tune the tradeoff parameter used by the Banditron algorithm. We showed through extensive experimental study that the proposed approaches are effective in adjusting the exploration-exploitation tradeoff. In particular, we found that banditron_ag2 and banditron_ag3 achieve similar or better performance compared to Banditron with the best value for $\gamma$. In the future, we plan to exploit other approaches for balancing the exploration-exploitation tradeoff other than the $\epsilon$-greedy strategy applied in Banditron. 
-- घ- - Banditron_Best

o....Banditron_worst

- $\diamond$ - Banditron_ag0

- - - Banditron_ag1

- +-Banditron_ag2

- -x-- Banditron_Ag3
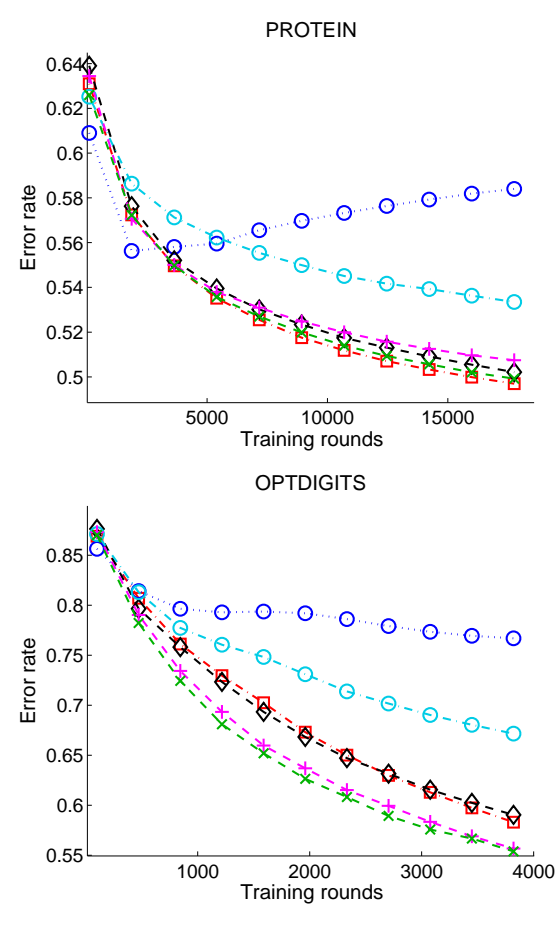

ABALONE

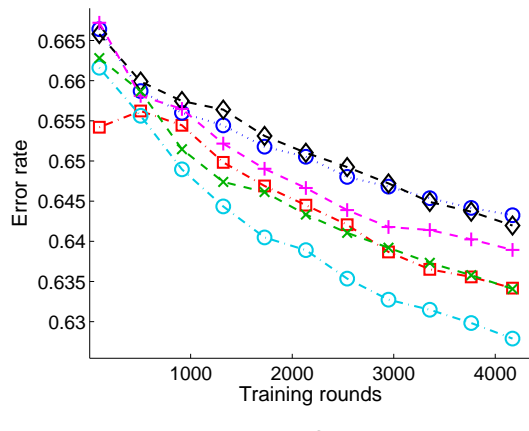

REDWINEQUALITY

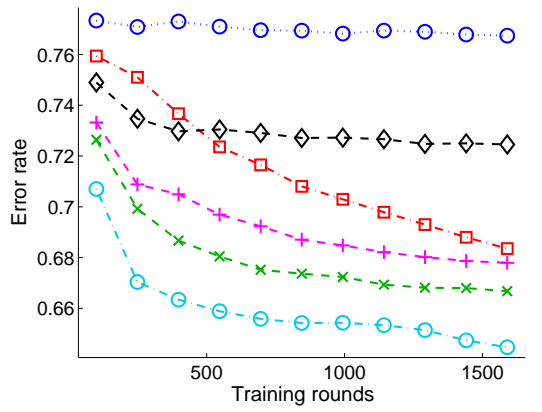

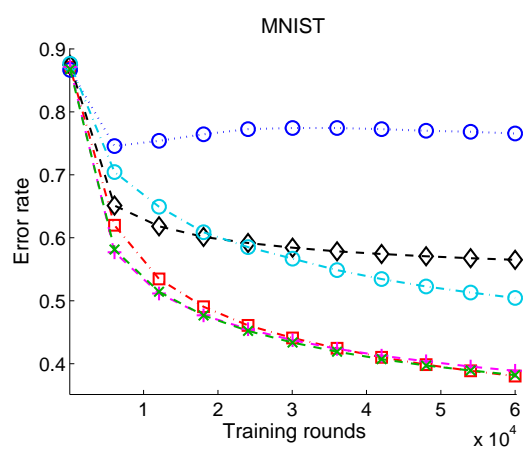
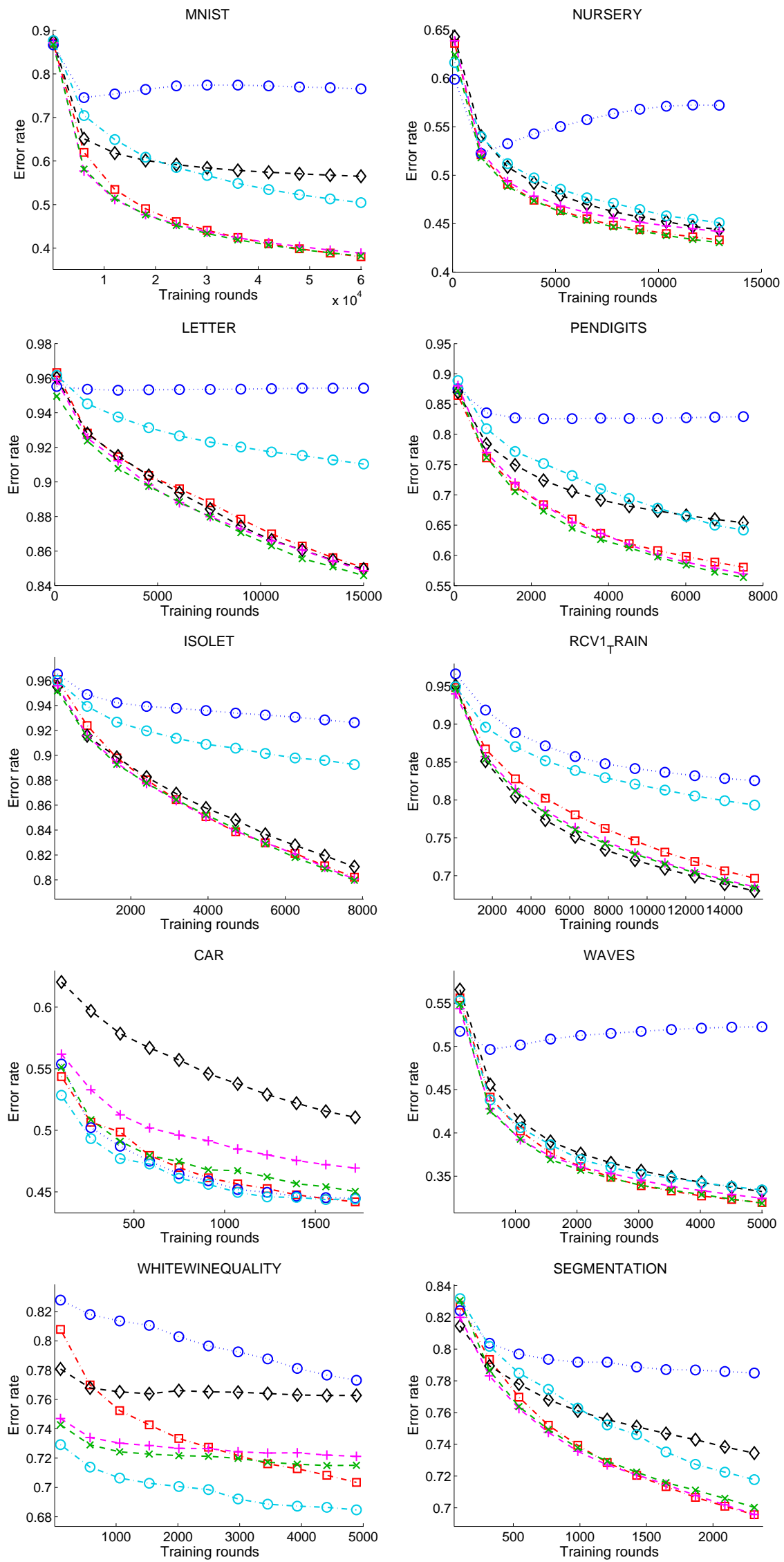

Figure 3: The error rates of different methods over trials. Each point on a curve is the average results of 50 randomly generated sequences of data. 


\section{ACKNOWLEDGEMENT}

This work is supported in part by National Science Foundation (IIS-0643494), and the Office of Navy Research (N0001409-1-0663).

\section{REFERENCES}

[1] Peter Auer, Nicolò Cesa-Bianchi, and Paul Fischer. Finite-time analysis of the multiarmed bandit problem. Machine Learning, 47(2-3):235-256, 2002.

[2] Alina Beygelzimer, John Langford, Lihong Li, Lev Reyzin, and Robert E. Schapire. An optimal high probability algorithm for the contextual bandit problem. Computational Research Repository, abs/1002.4058, 2010.

[3] N. Cesa-Bianchi and G. Lugosi. Prediction, learning, and games. Cambridge Univ Pr, 2006.

[4] Chih-Chung Chang and Chih-Jen Lin. Libsvm : a library for support vector machines, 2001.

[5] Eyal Even-Dar, Shie Mannor, and Yishay Mansour. PAC bounds for multi-armed bandit and markov decision processes. In COLT '02: Proceedings of the 15th Annual Conference on Computational Learning Theory, pages 255-270, 2002.

[6] Eyal Even-Dar, Shie Mannor, and Yishay Mansour. Action elimination and stopping conditions for the multi-armed bandit and reinforcement learning problems. Journal of Machine Learning Research, 7:1079-1105, 2006.

[7] A. Frank and A. Asuncion. UCI machine learning repository, 2010.

[8] Sham M. Kakade, Shai Shalev-Shwartz, and Ambuj Tewari. Efficient bandit algorithms for online multiclass prediction. In ICML 2008: Proceedings of the 25th international conference on Machine learning, pages 440-447, 2008.

[9] John Langford and Zhang Tong. The epoch-greedy algorithm for contextual multi-armed bandits. In NIPS 200\%: Proceeding of the 20th Annual Conference on Neural Information Processing System, 2007.
[10] D. D. Lewis, Y. Yang, T. Rose, and F. Li. Rcv1: A new benchmark collection for text categorization research. Journal of Machine Learning Research, 5:361-397, 2004.

[11] Lihong Li, Wei Chu, John Langford, and Robert E. Schapire. A contextual-bandit approach to personalized news article recommendation. In $W W W$ '10: Proceedings of the 19th international conference on World wide web, pages 661-670, New York, NY, USA, 2010. ACM.

[12] Wei Li, Xuerui Wang, Ruofei Zhang, Ying Cui, Jianchang Mao, and Rong Jin. Exploitation and exploration in a performance based contextual advertising system. In KDD 2010: Knoledge Discovery and Data Mining, pages 27-36, 2010.

[13] Shie Mannor and John N. Tsitsiklis. The sample complexity of exploration in the multi-armed bandit problem. Journal of Machine Learning Research, 5:623-648, 2004.

[14] Herbert Robbins. some aspects of the sequential design of experiments. Bulletin of the American Mathematical Society, 58:527-535, 1952.

[15] Herbert Robins. Some aspects of the sequential design of experiments. Bull. Amer. Math. Soc., 58(5):527-535, 2010.

[16] F. Rosenblatt. The perceptron: a probabilistic model for information storage and organization in the brain. Psychological review, 65:386-408, 1958.

[17] Joannès Vermorel and Mehryar Mohri. Multi-armed bandit algorithms and empirical evaluation. In In European Conference on Machine Learning, pages 437-448. Springer, 2005.

[18] Shijun Wang, Rong Jin, and Hamed Valizadegan. A potential-based framework for online multi-class learning with partial feedback. In ISTATS 2010: Artificial Intelligence and Statistics, 2010.

[19] C. Watkins. Learning from delayed Rewards. PhD thesis, Cambridge, 1989. 